\title{
The child with immune thrombocytopenia: to treat or not to treat, is that still the question?
}

\section{Nichola Cooper ${ }^{1}$ and Douglas B. Cines ${ }^{2}$}

${ }^{1}$ Centre for Haematology, Department of Medicine, Imperial College, Hammersmith Hospital, London, UK and ${ }^{2}$ Departments of Pathology and Laboratory Medicine and Medicine, University of Pennsylvania-Perelman School of Medicine, Philadelphia, PA, USA

E-mail: DOUGLAS B. CINES - dcines@pennmedicine.upenn.edu

doi:10.3324/haematol.2019.229179

I $\mathrm{n}$ this issue of the Journal, Tarantino et al. ${ }^{1}$ describe their experience with the use of romiplostim to treat children with persistent immune thrombocytopenia (ITP). The results of this study also have implications for management of children who present with ITP. ${ }^{1}$

Eighty to $90 \%$ of children who present with ITP go into remission within 12 months and life-threatening bleeding is rare. ${ }^{2,3}$ However, this means that $10-20 \%$ do not go into remission, and even those whose disease eventually remits can experience bleeding symptoms and limitations in quality of life until this occurs. There is considerable heterogeneity in symptomatology and responsiveness that makes treatment decisions challenging for patients, families and clinicians alike. ${ }^{4}$ ITP presenting in very young children, with a peak around 3-4 years, is somewhat more likely to be self-limiting, whereas ITP presenting in the teen years is more likely to follow the more prolonged course seen in adults. ${ }^{2}$ Treatment decisions are further complicated by the fact that standard therapies are associated with burdensome adverse effects. Steroids cause metabolic and behavior problems that limit dosing and duration of treatment. Intravenous immunoglobulin (IVIG) can cause severe headaches, requires children to miss school, and its beneficial effects are transient. Splenectomy and immunosuppressive treatment, including rituximab, are used with caution in children because of the potential for increased risk of infection in an immature immune system and the unknown potential for late effects such as secondary malignancies if immunosupressants are used persistently. In view of these adverse effects of treatment, the low incidence of internal bleeding, the high likelihood of remission, and the absence of data indicating an effect on durable remission, many guidelines recommend not treating children with ITP unless bleeding is severe (Figure 1, left). ${ }^{6,7}$ However, this clinical situation is not ideal. Episodic and unpredictable bleeding along with low platelet counts can lead to child and parental anxiety, restrictions on activities, and a significant negative impact on social and emotional development. Children with ITP and significant thrombocytopenia can have reduced school attendance, fail to participate in athletic activities, and families may be reluctant to travel far from home. ${ }^{5,8}$

Therefore, there is an unmet need for less toxic and less invasive approaches to managing children with: a) symptomatic persistent/chronic ITP; b) those at significant risk of bleeding based on platelet count and/or comorbidities; and c) those with impaired health care related quality of life (HRQol) due to the need for considerable medical attention or because of the emotional impact associated with bleeding, the fear of bleeding, and the side effects of current treatments.

Thrombopoietin receptor agonists (TRA) have been used successfully for these purposes in adults with ITP for approximately ten years. ${ }^{9}$ From $80 \%$ to $90 \%$ of adults show a response, almost all of which are durable with continued treatment. Response rates are even higher when crossovers from either agent to the other are included and less stringent response criteria, e.g. attaining a patient-specific hemostatic platelet count, are employed. There is now less concern over the risk of thrombosis and marrow fibrosis based on long-term safety studies, ${ }^{10,11}$ and $20-30 \%$ of adult patients are able to discontinue therapy by 2-3 years of treatment (reviewed by Ghanima et al. ${ }^{9}$ ).

Following upon from this track record in adults, it became important to assess whether similar efficacy and safety profiles would be seen in children with ITP. Smaller randomized studies showing efficacy and no major safety concerns led to the approval of romiplostim in 2018 for use in children with ITP who are over one year of age with ITP $\geq 6$ months duration. ${ }^{12,13}$ Treatment was associated with improvement in child self-reported HRQol and reduced parental burden. ${ }^{14}$ However, there continued to be a need to establish whether efficacy was sustained, to investigate the long-term safety, and to assess the impact of romiplostim on the natural history of ITP in children.

In this issue, Tarantino et al. report the outcomes of an open-label extension study of romiplostim in 65 children with ITP of $\geq 6$ months duration, ${ }^{1} 15$ of whom were receiving this treatment for the first time. Median duration of disease was 3 years (range: 1-13 years) and median baseline age was 11 years (range: 3-18 years). Median duration of treatment was 2.6 years (range: 1-7 years), median average weekly dose $4.8 \mu \mathrm{g} / \mathrm{kg}$ (but was as low as $0.1 \mu \mathrm{g} / \mathrm{kg}$ in some), and the median response rate using previously published strict criteria $(72 \%$ at $\geq 75 \%$ of visits; $58 \%$ at $\geq 90 \%$ of visits) was comparable to outcomes in adults. Fifty-nine (91\%) of the 65 children or their caregivers "self-administered" treatment at home at least once starting at median study week 7 . Fifty-seven reported miscellaneous bleeding adverse effects (AE) as would be expected in a trial in this population, but no thrombosis or other major drug-related AE were identified, although bone marrow examinations that might have revealed fibrosis were performed in only two subjects. Of note, 23 (35\%) required rescue treatment on at least one occasion. Also, 29 (44\%) left the study for a variety of reasons, including withdrawal of consent for unspecified reasons, non-compliance, need for other treatment, and two for AE unrelated to treatment. Treatment-free responses were observed in 15 children (23\%) who had ITP for a median of four years (range: 1-12 years), had received romiplostim for two years (range: 1-6 years), and the response was maintained in 14 for a median of one 


\section{Time for new approach to early management of children with ITP?}

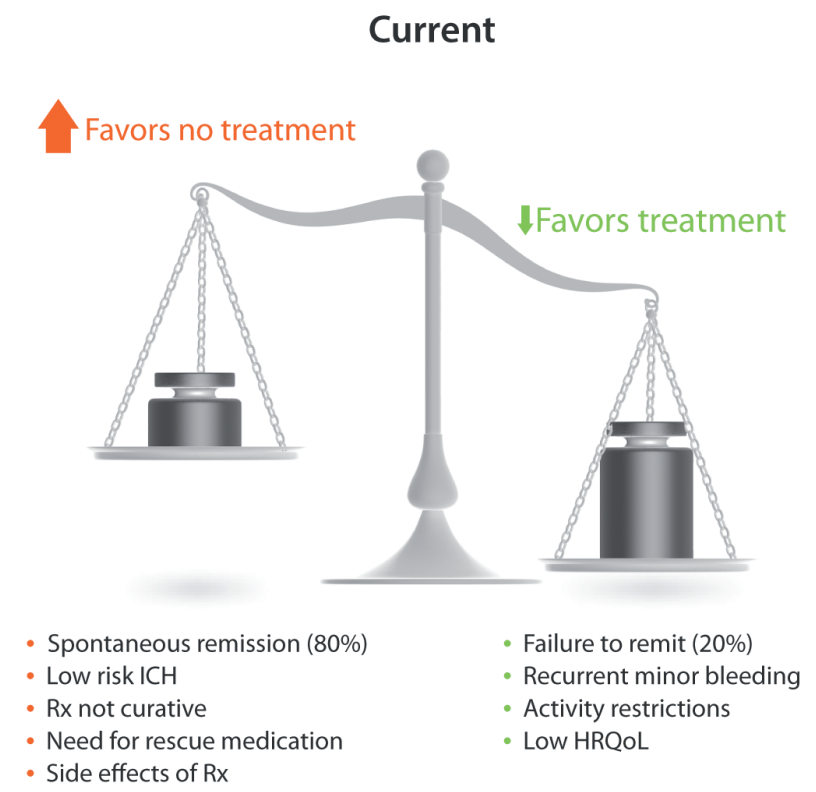

Favors no treatment
Advent of TRAs

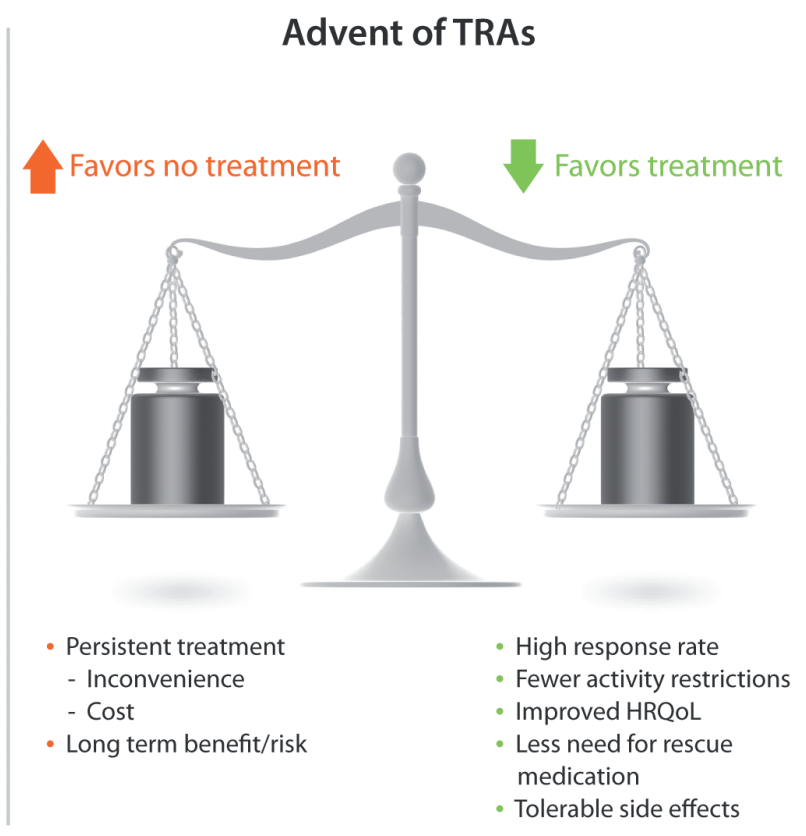

Figure 1. (Left) Currently, children who present with immune thrombocytopenia (ITP) are followed without therapy in the absence of significant bleeding. Life-threatening bleeding is rare, remission is common, and no therapy has been shown to alter the natural history of the disorder. The decision not to treat is therefore dominated by the side effects of therapy. The side effects of corticosteroids and intravenous immunoglobulin (IVIG) and the risk of infection after splenectomy and perhaps rituximab mitigate in favor of observation. Indeed, treatment may worsen health care related quality of life (HRQol). (Right) With the advent of thrombopoietin receptor agonists (TRA), the proper balance has become less clear. TRA provide a therapeutic option with a high response rate and fewer concerns about side effects, as described in Tarentino et al. ${ }^{1}$ Improvement in platelet counts may reduce nuisance bleeding and the need for rescue therapy and hospital attendance, increase participation in physical and social activities, reduce anxiety around low platelet counts, and improve HRQoL. ICH: intracranial hemorrhage; Rx: treatment.

year (range: 0.4-2.1 years); younger age at first dose was associated with treatment-free responses.

This is the most comprehensive placebo-controlled study of TRA in pediatric ITP. The data show that romiplostim is a highly effective maintenance therapy for children with ITP of at least six months duration who did not respond to, or perhaps were intolerant of, prior therapies and who experienced bleeding or are at risk of bleeding because of low platelet counts. Romiplostim was well tolerated, no significant drug-related AE were observed at two years of study, and, importantly, treatment could be given at home by trained patients or family members. The high rate of withdrawal complicates delineating sustained reduction in bleeding events, use of concurrent medications, and the need for rescue interventions that might occur with more extended use in practice. Also, the implication that romiplostim induces remission is less compelling in the absence of a control population.

Eltrombopag has also been approved for use in the pediatric population in a similar setting and with similar outcomes. ${ }^{15-18}$ Iron deficiency and transaminitis have been reported in some children treated with eltrombopag. ${ }^{16,19}$ One as yet theoretical concern when contemplating longterm use is that eltrombopag acts on bone marrow stem cells as well as on megakaryocytes. ${ }^{20}$ In the absence of a head-to-head study, the choice between agents often comes down to the requirement for dietary restrictions and daily administration with eltrombopag versus parenteral administration with more frequent medical visits with romiplostim, as well as differences in cost and insurance coverage.

One important issue raised by this study is whether consideration should be given to starting romiplostim or eltrombopag in children with ITP without the need to wait six months from diagnosis. This is particularly relevant for children who experience extensive bruising, epistaxis, menorrhagia, or other symptoms that require frequent medical interventions or that lead to loss of school days, withdrawal from competitive sports or otherwise reduce HRQol (Figure 1, right). If durable safety is established, TRA may also be advantageous for those with less severe symptoms but significant anxiety related to low platelet counts. Indeed, this trend in treatment is becoming apparent, as approximately $20 \%$ of the children treated with romiplostim in the ICON2 analysis had severe, refractory, but newly diagnosed ITP, and a few were treated to resume physical activities or to improve QoL. ${ }^{17}$ Improvements in platelet count and HRQol have been reported with this approach, although the number of patients studied is small and the duration of follow up is short. ${ }^{18,21}$

In addition to continued surveillance relating to safety in the pediatric population, future studies are needed to determine if initiating treatment earlier in the disease reduces bleeding, the need for other treatment, medical visits, and lost school days while improving the emotional well-being of the child and the family. Therefore, studies such as the one reported here by Tarantino et al. could 
have a major impact on the way in which pediatric hematologists approach the long standing "To treat or not to treat" question when evaluating children with suspected ITP at the time of or soon after presentation ${ }^{22}$ (Figure 1). This study also highlights a compelling need for careful study of children now receiving TRA for other indications, including hereditary thrombocytopenia and associated qualitative platelet disorders, neonatal thrombocytopenia, and thrombocytopenia following chemotherapy or bone marrow transplantation (reviewed by Neunert and $\operatorname{Rose}^{23}$ ).

\section{References}

1. Tarantino MD, Bussel JB, Blanchette VS, et al. Long-term treatment with romiplostim and treatment-free platelet responses in children with chronic immune thrombocytopenia. Haematologica. 2019; 104(11):2283-2291.

2. Bennett CM, Neunert C, Grace RF, et al. Predictors of remission in children with newly diagnosed immune thrombocytopenia: Data from the Intercontinental Cooperative ITP Study Group Registry II participants. Pediatr Blood Cancer. 2018;65(1)

3. Neunert CE, Buchanan GR, Imbach P, et al. Bleeding manifestations and management of children with persistent and chronic immune thrombocytopenia: data from the Intercontinental Cooperative ITP Study Group (ICIS). Blood. 2013;121(22):4457-4462.

4. Shaw J, Kilpatrick K, Eisen M, Tarantino M. The incidence and clinical burden of immune thrombocytopenia in pediatric patients in the United States. Platelets. 2019:1-8.

5. Cooper N. A review of the management of childhood immune thrombocytopenia: how can we provide an evidence-based approach? Br J Haematol. 2014;165(6):756-767.

6. Provan D, Stasi R, Newland AC, et al. International consensus report on the investigation and management of primary immune thrombocytopenia. Blood. 2010;115(2):168-186.

7. Neunert C, Lim W, Crowther M, Cohen A, Solberg L Jr, Crowther MA; American Society of Hematology. The American Society of Hematology 2011 evidence-based practice guideline for immune thrombocytopenia. Blood. 2011;117(16):4190-4207.

8. Klaassen RJ, Blanchette V, Burke TA, et al. Quality of life in childhood immune thrombocytopenia: international validation of the kids' ITP tools. Pediatr Blood Cancer. 2013;60(1):95-100.

9. Ghanima W, Cooper N, Rodeghiero F, Godeau B, Bussel JB. Thrombopoietin receptor agonists: ten years later. Haematologica. 2019;104(6):1112-1123.
10. Saleh MN, Bussel JB, Cheng G, et al. Safety and efficacy of eltrombopag for treatment of chronic immune thrombocytopenia: results of the long-term, open-label EXTEND study. Blood. 2013;121(3):537545.

11. Cines DB, Gernsheimer T, Wasser J, et al. Integrated analysis of longterm safety in patients with chronic immune thrombocytopaenia (ITP) treated with the thrombopoietin (TPO) receptor agonist romiplostim. Int J Hematol. 2015;102(3):259-270.

12. Bussel JB, Buchanan GR, Nugent DJ, et al. A randomized, doubleblind study of romiplostim to determine its safety and efficacy in children with immune thrombocytopenia. Blood. 2011;118(1):28-36.

13. Tarantino MD, Bussel JB, Blanchette VS, et al. Romiplostim in children with immune thrombocytopenia: a phase 3, randomised, double-blind, placebo-controlled study. Lancet. 2016;388(10039):45-54.

14. Mathias SD, Li X, Eisen M, Carpenter N, Crosby RD, Blanchette VS A Phase 3, Randomized, Double-Blind, Placebo-Controlled Study to Determine the Effect of Romiplostim on Health-Related Quality of Life in Children with Primary Immune Thrombocytopenia and Associated Burden in Their Parents. Pediatr Blood Cancer. 2016;63(7):1232-1237.

15. Tumaini Massaro J, Chen Y, Ke Z. Efficacy and safety of thrombopoietin receptor agonists in children with chronic immune thrombocytopenic purpura: meta-analysis. Platelets. 2019;30(7):828-835.

16. Kim TO, Despotovic J, Lambert MP. Eltrombopag for use in children with immune thrombocytopenia. Blood Adv. 2018;2(4):454-461.

17. Neunert C, Despotovic J, Haley K, et al. Thrombopoietin Receptor Agonist Use in Children: Data From the Pediatric ITP Consortium of North America ICON2 Study. Pediatr Blood Cancer. 2016;63 (8):1407-1413.

18. Grainger JD, Blanchette VS, Grotzinger KM, Roy A, Bussel JB. Health-related quality of life in children with chronic immune thrombocytopenia treated with eltrombopag in the PETIT study. $\mathrm{Br}$ J Haematol. 2019;185(1):102-106.

19. Lambert MP, Witmer CM, Kwiatkowski JL. Therapy induced iron deficiency in children treated with eltrombopag for immune thrombocytopenia. Am J Hematol. 2017:92(6):E88-E91.

20. Kao YR, Chen J, Narayanagari SR, et al. Thrombopoietin receptorindependent stimulation of hematopoietic stem cells by eltrombopag. Sci Transl Med. 2018;10(458).

21. Grace RF, Shimano KA, Bhat R, et al. Second-line treatments in children with immune thrombocytopenia: Effect on platelet count and patient-centered outcomes. Am J Hematol. 2019;94(7):741-750.

22. Neunert CE, Cooper N. Evidence-based management of immune thrombocytopenia: ASH guideline update. Hematology Am Soc Hematol Educ Program. 2018:2018(1):568-575.

23. Neunert CE, Rose MJ. Romiplostim for the management of pediatric immune thrombocytopenia: drug development and current practice. Blood Adv. 2019;3(12):1907-1915. 